\title{
Effect of partial Ti substitution at Zn sites on the Structural, Electronic and Magnetic Properties of Zn3P2
}

\author{
Jaiganesh Gnana Sekaran ${ }^{1}$ and Mathi Jaya $S^{1}$ \\ ${ }^{1}$ IGCAR
}

November 6, 2020

\begin{abstract}
Using the ab-initio calculations based on density functional theory, we have investigated the structural, electronic and magnetic properties of Ti-substituted Zn3P2 compound. One and two Ti atom replacements in the unit cell of Zn3P2, which contain eight molecules per formula unit (40 atoms), are considered in the study. Our results show that the ferromagnetic phase is favored for the single $\mathrm{Ti}$ atom substitution, as the total energy corresponding to the ferromagnetic phase is lower than that of the nonmagnetic phase. A considerable value of the magnetic moment at the Ti site is obtained from our calculations.
\end{abstract}

\section{Hosted file}

Jaiganesh_Manuscript.pdf available at https://authorea.com/users/373715/articles/491324effect-of-partial-ti-substitution-at-zn-sites-on-the-structural-electronic-and-magneticproperties-of-zn3p2 